Check for updates

Cite this: RSC Adv., 2017, 7, 36048

Received 27th April 2017

Accepted 18th June 2017

DOI: 10.1039/c7ra04712f

rsc.li/rsc-advances

\title{
Novel imprinted polyethyleneimine nano- fluorescent probes with controllable selectivity for recognizing and adsorbing metal ions $\uparrow$
}

\begin{abstract}
Jin Jia, $\dot{t}^{\text {acd }}$ Gui-Juan Cheng, $\dot{t}^{\mathrm{b}}$ Aihua Wu (D) *c and Shengji Luan ${ }^{\mathrm{a}}$
Novel ion imprinted nano-fluorescent probes (IIP-fluorescein) based on polyethyleneimine (PEI) were obtained via simple imprinting and grafting with fluorescein, and used for high recognition, detection and adsorption of $\mathrm{Cu}^{2+}, \mathrm{Zn}^{2+}$ and $\mathrm{Cr}^{3+}$ in aqueous solution. The selectivity of $\mathrm{PEI}$ is adjustable through an imprinting process for $\mathrm{Cu}^{2+}, \mathrm{Zn}^{2+}$ or $\mathrm{Cr}^{3+}$, which have a strong affinity for the amino group. Each probe reveals specific selectivity to and recognition of the relevant template metal ion over seven other common metal ions. The three probes exhibit a large linear detection range, high accuracy and strong adsorption capacity for $\mathrm{Cu}^{2+}, \mathrm{Zn}^{2+}$ and $\mathrm{Cr}^{3+}$. The sensitive and selective detection, as well as the removal of $\mathrm{Cu}^{2+}, \mathrm{Zn}^{2+}$ and $\mathrm{Cr}^{3+}$ by using IIP-fluorescein was further demonstrated in polluted tap and river water, indicating their potential application in environmental monitoring and treatment. Additionally, the sensing mechanism of IIP-fluorescein for metal ions was investigated through Gaussian 09. The synergistic effect of photo-induced electron transfer (PET) and intramolecular charge transfer (ICT) was demonstrated in the sensing process for metal ions. The design concept might propose a method for developing and studying other fluorescent probes with similar systems (containing amino groups, mercapto groups or cyano groups).
\end{abstract}

\section{Introduction}

The detection and control of metal ions in the aqueous environment remains a major issue because of their high toxicity, persistence and susceptible carcinogenic effect. ${ }^{1-4}$ Selective recognition of metal ions has become an indispensable task for a wide range of applications related to chemical, biological, medicinal and environmental sciences. Because of the high sensitivity, high speed and cheap instrumentation, in particular the fluorescence sensors and switches have been actively investigated. ${ }^{5-7}$

Up to now, extensive research has been conducted on fluorescent probes with aminoquinoline, ${ }^{8}$ porphyrin, ${ }^{9}$ coumarin, ${ }^{10}$ rhodamine derivatives ${ }^{11}$ and polycyclic aromatic hydrocarbon, ${ }^{12}$

${ }^{a}$ College of Environmental Science and Engineering, Peking University, Beijing 100871, China

${ }^{b}$ Key Laboratory of Computational Chemistry and Drug Design, College of School of Chemical Biology and Biotechnology, Peking University Shenzhen Graduate School, Shenzhen 518055, China

'Key Laboratory of Environment Simulation and Pollution Control, PKU-HKUST Shenzhen-Hongkong Institution, Shenzhen 518057, China. E-mail: phenix_wah@ aliyun.com

${ }^{d}$ Shenzhen Graduate School of Harbin Institute of Technology, Shenzhen 518055, China

$\dagger$ Electronic supplementary information (ESI) available. See DOI: $10.1039 / \mathrm{c} 7 \mathrm{ra04712f}$

$\ddagger$ These authors contributed equally to this work. etc. However, most of those small-molecule fluorescent probes display the disadvantage of anti-jamming, stability, reusability and causing secondary pollution. To overcome these weaknesses, researchers made great efforts to develop various novel probes and methods, including quantum dots, ${ }^{13-15}$ biosensors ${ }^{16-18}$ and nano-fluorescent probes. ${ }^{19-21}$ The nano-fluorescent probes are extremely attractive and arouse the interest of researchers. It shows more stable, easy recovery and higher sensitivity due to the huge surface area per unit mass ( $\mathrm{S} / \mathrm{M}$ ratio). ${ }^{22,23}$ The nanofluorescent probes could be easily designed by various receptor and fluorophore. However the reversal design of fluorescent probes according to different target metal ions is still a big problem. Thus it is very necessary but also challenging to develop method for fabricating probes and sensing target metal ions.

Ion imprinting could be a promising solution to that problem since its controllable selectivity. The ion imprinted polymer (IIP) can be conveniently synthesized according to the target metal ions, and shows advantage of anti-interference and stability. ${ }^{24-26}$ Recently, many studies focus on the applications of fluorescent probes grafting with IIP. ${ }^{27-29}$ However, little attempt was done on selectivity control mechanism.

Polyethyleneimine (PEI) is a cationic polymer with large amount of amine groups. Thus, it can form chelation complexes with heavy metal ions possessing good hydrophilicity, high adsorption capacity and rapid chelating rate. ${ }^{30-32}$ In our previous work, PEI revealed strong affinity towards $\mathrm{Cu}^{2+}, \mathrm{Zn}^{2+}$, $\mathrm{Cr}^{3+}$, and also showed great potential of specific selectivity. ${ }^{33} \mathrm{To}$ 
further investigate the control mechanism of selectivity, ion imprinted nano-fluorescent probes based on PEI were achieved via simple imprinting by $\mathrm{Cu}^{2+}, \mathrm{Zn}^{2+}$ or $\mathrm{Cr}^{3+}$ and grafting with fluorescein. The selectivity could be further enhanced and specialized by imprinting effect and fluorescence signal transmission. The probe imprinted by $\mathrm{Cu}^{2+}, \mathrm{Zn}^{2+}$ or $\mathrm{Cr}^{3+}$ respectively reveals specific selectivity and recognition to the relevant template metal ion over seven other common metal ions. Additionally, the sensing mechanism for metal ions was investigated through Frontier molecular orbital and electron density distribution. The novel probes were applied as sensor and absorbent for metal ions in aqueous solution. The three probes exhibit large linear detection range, high accuracy and strong adsorption capacity for $\mathrm{Cu}^{2+}, \mathrm{Zn}^{2+}$ and $\mathrm{Cr}^{3+}$. The sensitive and selective detection, as well as the removal of $\mathrm{Cu}^{2+}, \mathrm{Zn}^{2+}$ and $\mathrm{Cr}^{3+}$ by using IIP-fluorescein was further demonstrated in polluted tap and river water, indicating their potential application in environmental monitoring and treatment. The design concept might propose a method for developing and studying other fluorescent probes with similar systems.

\section{Experimental section}

\subsection{Chemicals and apparatus}

Branched polyethyleneimine (PEI) with weight average molecular weight of $25 \mathrm{kDa}$ and epichlorohydrin $(\mathrm{ECH})$ was obtained from Aldrich. Ammonia, diethyl ether and ethyl acetate was obtained from Guangzhou Chemical Reagent Factory. Fluorescein, hydroxybenzotriazole (HOBT), $N, N, N,{ }^{\prime} N^{\prime}$-tetramethyluronium hexafluorophosphate (HBTU), $N, N$-diisopropylethylamine (DIEA), copper nitrate trihydrate and other several kinds of metal salts were received from Sinopharm Chemical Reagent Corporation. All those chemical reagents used in this study were of analytical grade and were used without further purification. Freshly deionized and distilled water was used as the dispersion medium throughout the experiments.

Fluorescence spectrum was recorded using Hitachi fluorescence spectrophotometer (F-4600). Flame atomic absorption spectrophotometer (FAAS) was purchased from Thermo (SolaarS4). Scanning electron microscope (SEM) was from Phenom (Pro). Fourier transform infrared spectrometer (FTIR) was from Thermo (Nicolet IS50). Laser particle size analyzer was form Malvern (Nano ZS90). Filtering centrifuge tube from Millipore (50 mL, MWCO 3k) and dialysis tube from Yuanye BioTechnology Corporation (MWCO 8000-14 000, 100k) were utilized in this study.

\subsection{Preparation of metal ion imprinted PEI-fluorescein nano-fluorescent probes}

Heavy metal ion imprinted PEI nanoparticles were synthesized according to our previous work. ${ }^{33} 1 \mathrm{~mol}$ of $\mathrm{NH}_{4} \mathrm{OH}$ (aqueous solution) was added drop-wise to $2 \mathrm{~mol}$ of epichlorohydrin at $60{ }^{\circ} \mathrm{C}$. The oligomer epichlorohydrin amine (ECHA) was obtained under vigorous agitation for $3 \mathrm{~h} .3 .025 \mathrm{~g}$ of $\mathrm{Cu}\left(\mathrm{NO}_{3}\right)_{2} \cdot 3 \mathrm{H}_{2} \mathrm{O}$ were dissolved in $30 \mathrm{~mL}$ of water and mixed with $5 \mathrm{~g}$ PEI. The mixture was under ultrasound for $30 \mathrm{~min}$ to prepare the desired complexes. Then, the obtained oligomer ECHA was added dropwise with constant stirring at $80^{\circ} \mathrm{C}$. $30 \mathrm{~min}$ after finishing adding of ECHA, $1 \mathrm{M} \mathrm{NaOH}$ was added to adjust solution $\mathrm{pH}$ to 10 . The whole reaction mixture was stirred at $80^{\circ} \mathrm{C}$ for $3 \mathrm{~h}$. Afterward the copper ions were eluted repeatedly with $1 \mathrm{M}$ hydrochloric acid and EDTA. The metal ions were recovered completely as confirmed by the conductivity of solution achieving stability. Finally the copper ion imprinted PEI nanoparticles (Cu-IIP) were washed with extensive distilled water until the washing water was neutral and stored at sealed bottle for further use. Also Zn-IIP, Cr-IIP and non-imprinted polymer (NIP) was synthesized by adopting the same protocols as above.

The nano-fluorescent probes were prepared by grafting fluorescein onto surface of IIP. $0.166 \mathrm{~g}$ fluorescein was dissolved in $50 \mathrm{~mL}$ DMF, following by adding of HOBT, HBTU and DIEA (mole ratio of $1: 1: 2$ ) with stirring for $3 \mathrm{~h}$. Then the solution was mixed with previously obtained IIP with stirring at room temperature. After standing for $12 \mathrm{~h}$, the mixed solution was extracted by diethyl ether and washed repeatedly with ethyl acetate. To remove unreacted fluorescein, the products were dialyzed (MWCO 8000-14 000) with ethanol/water solution and confirmed by fluorescence spectrophotometer, then dialyzed with distilled water for 3 days. Finally, IIP grafted fluorescein nano-fluorescent probes (M-IIP-fluorescein) were obtained and stored at $4{ }^{\circ} \mathrm{C}$ for future usage.

\subsection{Morphological and spectral characterization of M-IIP- fluorescein}

Typical scanning electron microscopy observations (choose $\mathrm{Cu}-$ IIP-fluorescein as example) show the nanoscale of fluorescent probes (Fig. 1). A great difference of morphology between $\mathrm{Cu}-$ IIP-fluorescein (A) and NIP-fluorescein (B) is found and reveal the imprinting effect of metal ions. The $\mathrm{Cu}$-IIP-fluorescein shows uniform shape of quadrahedron crystals with diameters around $190 \mathrm{~nm}$, while NIP-fluorescein displays irregular shape and varying diameters of nanoparticle. The imprinting of metal ions improves the formation of metal complex and immobilizes the crystals structure in the synthesis process of fluorescent probes. The details of the particle diameter, zeta potential and isoelectric point are given in Table S1 in the ESI. $\dagger$

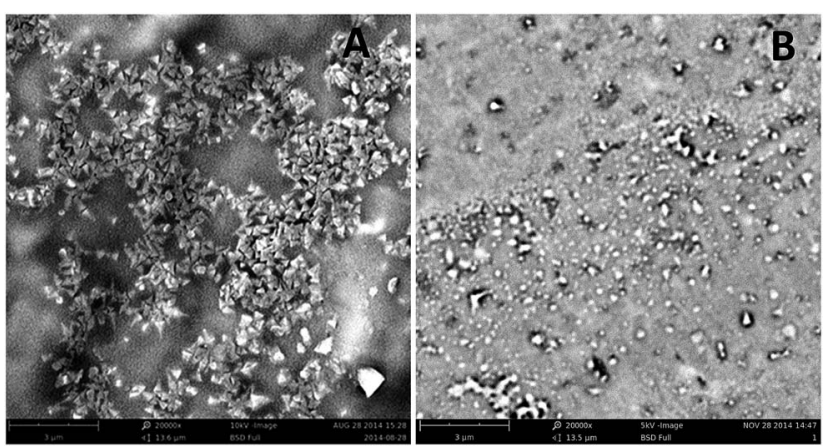

Fig. 1 SEM observations of nano-fluorescent probes: (A) Cu-IIPfluorescein and (B) NIP-fluorescein. 
To characterize chemical composition of fluorescent probes, infrared spectra of PEI, Cu-IIP, fluorescein and $\mathrm{Cu}$-IIP-fluorescein are performed (Fig. $\mathrm{S} 1 \dagger$ ). The characteristic peaks of PEI $\left(\mathrm{NH}^{4+}\right.$ at $2948 \mathrm{~cm}^{-1}$ and $\left.2840 \mathrm{~cm}^{-1}\right)$, IIP $\left(\mathrm{C}-\mathrm{O}\right.$ at $\left.1033 \mathrm{~cm}^{-1}\right)$ and fluorescein (phenols $\mathrm{C}-\mathrm{O}$ at $1213 \mathrm{~cm}^{-1}$ ) appearing in M-IIPfluorescein demonstrate that fluorescein has been successfully grafted onto surface of M-IIP.

\subsection{Procedures for metal ions detection and recovery}

In the present strategy, the $\mathrm{pH}$ and temperature played crucial roles for the detection sensitivity (Fig. S2 $\dagger$ ). Taking into account operational convenience, HAc-NaAc buffer solution $(0.1 \mathrm{M}, \mathrm{pH}$ 5.65) was applied to keep stable $\mathrm{pH}$ which had non-effect towards fluorescent probe or metal ions. Room temperature $\left(20^{\circ} \mathrm{C}\right)$ was selected as operational temperature for all experiments. Fluorescein, NIP-fluorescein and M-IIP-fluorescein were dissolved in HAc-NaAc buffer separately to prepare $0.1 \mu \mathrm{M}$ solution. Then, $1 \mathrm{mg} \mathrm{L}^{-1}$ of different metal ions were added to each solution. The fluorescence spectra of probes with different metal ions were monitored after the completion of hybrid reaction. For the adsorption and recovery experiment, $\mathrm{Cu}-\mathrm{IIP}-$ fluorescein, $\mathrm{Zn}$-IIP-fluorescein and Cr-IIP-fluorescein were successively added into mixed metal ions and filtered through ultrafiltration centrifuge tube (MWCO $3 \mathrm{k}$ ). The residue metal ions left in the filtrate were analyzed by an atomic adsorption spectrometer. The metal ion salts are as follows: $\mathrm{Cu}\left(\mathrm{NO}_{3}\right)_{2}$, $\mathrm{ZnCl}_{2}, \mathrm{CrCl}_{3}, \mathrm{Ni}\left(\mathrm{NO}_{3}\right)_{2}, \mathrm{CoCl}_{2}, \mathrm{~Pb}\left(\mathrm{NO}_{3}\right)_{2}, \mathrm{CdCl}_{2}$ and $\mathrm{AgNO}_{3}$. The river water samples were taken from Dasha River in Shenzhen.

\subsection{Computational details}

IIP of M-IIP-fluorescein and PEI were simplified into micromolecule containing 6 amino groups (ratio of primary amine, secondary amine and tertiary amine is $1: 1: 1)$. All the calculations were carried out with the Gaussian 09 package. ${ }^{34}$ The ground state structures were optimized using density functional theory (DFT) with the M06 hybrid functional. The LANL2DZ basis set with ECP was used for metal atoms and the 6-31G(d) basis set was used for other atoms. Frequency analysis was conducted at the same level of theory to verify the stationary points to be real minima and to obtain the thermodynamic energy corrections. Single point energies were calculated at the M06/SDD-6-311++G (d, p) level in water by using the SMD solvation model. The excited state related calculations were performed with the time dependent DFT (TD-DFT) at the M06/ SDD-6-311++G (d, p) level in water with the optimized structure of the ground state. The energies are presented in $\mathrm{kcal} \mathrm{mol}^{-1}$.

\section{Results and discussion}

\subsection{Fluorescence property and sensing scheme of fluorescent probes}

In the present study, we fabricated 3 kinds of imprinted nanofluorescent probes for highly sensitive and selective detection of target imprinted metal ions $\left(\mathrm{Cu}^{2+}, \mathrm{Zn}^{2+}\right.$ and $\left.\mathrm{Cr}^{3+}\right)$. Both probes contain two parts: the imprinted PEI nanoparticle in the inner core and grafted with fluorescein around. Fig. 2A schematically showed the sensing mechanism of template metal ions. The imprinted PEI acted as recognition moiety (electron donor), the fluorescein acted as the report moiety (electron acceptor), and they were linked with amido bond. When fluorescein grafted with IIP or NIP, the conjugated amino chains were introduced into the molecule, enhanced density of electron cloud and conjugation effect. In this case, it reveals a great increase of fluorescence intensity, as well as a $10 \mathrm{~nm}$ red shift (Fig. 2B). When metal ions presented in the aqueous solution, the coordination between IIP and target template metal ions $\left(\mathrm{Cu}^{2+}, \mathrm{Zn}^{2+}\right.$ or $\mathrm{Cr}^{3+}$ ) will occur. The lone pair electrons of amino group were occupied by the metal ions to form $\mathrm{M}-\left(\mathrm{NH}_{2}\right)_{n}$ complexes, which diminished or cut off electron donation ability towards fluorescein. As the result, the fluorescence intensity of probes was decreased. Otherwise, a control experiment was used to investigate if metal ions had an effect on the fluorescein, in which metal ions were added to a solution only containing fluorescein. The results indicated that metal ions made negligible contribution toward quenching the fluorescence of fluorescein (Fig. S3 $\dagger$ ).

The specific sensing mechanism was demonstrated with Frontier molecular orbital and electron density distribution by Gaussian 09. The Frontier molecular orbital distributions and energy of $\mathrm{Cu}$-IIP-fluorescein were presented in Fig. S4. $\dagger$ The orbital energy of IIP (HOMO-1) is lower than fluorescein (HOMO and LUMO) when absence of copper ions, which means PET process will not occur. When $\mathrm{Cu}$-IIP-fluorescein coordinates with copper ions, two types of electron transition $(\alpha$ electron and $\beta$ electron) will exist due to an unpaired electron in copper ions (II). The orbital and energy distributions of $\alpha$ electron are similar with absence of copper ions. On the contrary, LUMO lies on IIP for $\beta$ electron. The transition type on fluorescein is $\mathrm{HOMO} \rightarrow \mathrm{LUMO}+1$. This means orbital energy of IIP lies between transition energy of fluorescein. Therefore the PET process from fluorescein (LUMO+1) to IIP (LUMO) will occur.

To further verify the PET process, we calculated the vertical excitation results of $\mathrm{Cu}$-IIP-fluorescein in Table 1 . The evaluation of the electronic excited states and the photophysical properties of the probes are based on the selection rule for electronic transitions. ${ }^{35-37}$ The parameter to evaluate the possibility of a transition is the oscillator strength $(f)$. Usually transitions with $f>0.01$ are allowed; conversely, a small $f<0.01$ infers a forbidden transition. The same rules are applicable to the emission processes. ${ }^{38,39}$ In absence of copper ions, the value of $f(0.4023)$ indicates effective transition and fluorescence emission. After coordinating with copper ions, the transition is mainly composed by $\beta$ electron transition, however, the small $f$ (0.0014) reveals fluorescence quenching by PET process.

To investigate and interpret the ICT process, the electron density distribution of probes was calculated and presented in Fig. S5. $\uparrow$ The carbonyl on the fluorescein is electron-acceptor with electron density of -0.13 e. After grafting with IIP, the electron density distribution of probe is more homogeneous due to the electron donating effect and conjugation effect of IIP. The electron negativity of carbonyl is decreased with electron density of -0.118 e. In the meanwhile, the conjugation effect leads to decrease of probe dipole moment, followed by declining of energy gaps between excited state and ground state. As the result, the red 

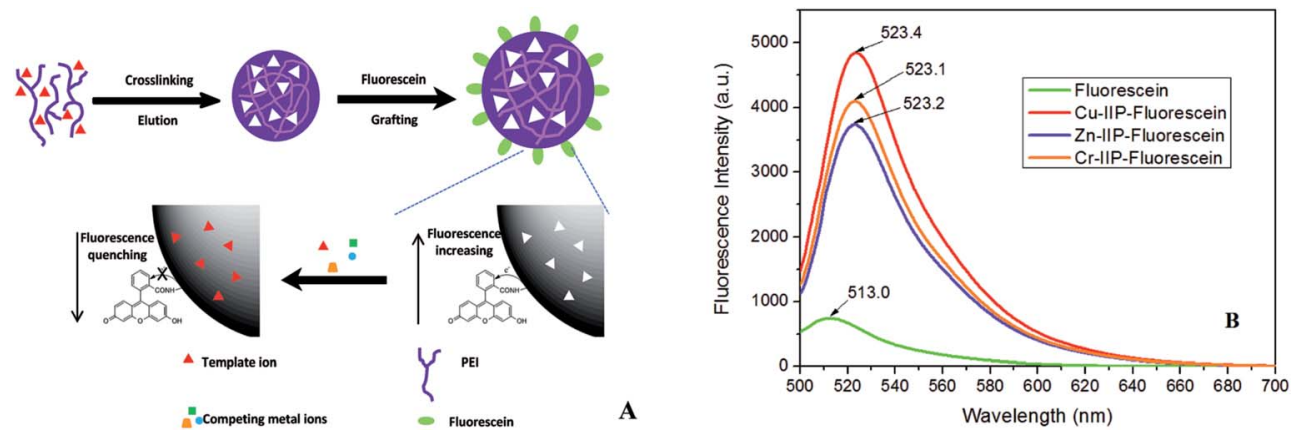

Fig. 2 (A) Schematic description of synthesis process of imprinted nano-fluorescent probes and sensing mechanism towards template metal ions. When fluorescein grafts with IIP or NIP, the fluorescence intensity is enhanced by introducing conjugated amino chains and electrondonating process. The probes could specifically recognize and coordinate with template metal ions, which will diminish or cut off electron donation ability towards fluorescein, leading the decrease of fluorescence intensity. (B) Fluorescence spectrum of fluorescein and nano-fluorescent probes $(0.1 \mu \mathrm{M})$

Table 1 Vertical excitation results of $\mathrm{Cu}-\mathrm{IIP}$-fluorescein before and after coordinating with copper ions

\begin{tabular}{|c|c|c|c|c|c|}
\hline Molecule & Electronic transition $^{a}$ & Energy $(\mathrm{eV})$ & $f^{b}$ & Composition $^{c}$ & $\mathrm{CI}^{d}$ \\
\hline $\mathrm{Cu}$-IIP-fluorescein & $\mathrm{S}_{0} \rightarrow \mathrm{S}_{1}$ & 2.9391 & 0.4023 & $\begin{array}{l}\mathrm{H} \rightarrow \mathrm{L} \\
\mathrm{H}-2 \rightarrow \mathrm{L}\end{array}$ & $\begin{array}{l}0.67601 \\
0.10973\end{array}$ \\
\hline
\end{tabular}

${ }^{a}$ Only $\mathrm{S}_{1}$ excited states were considered. ${ }^{b}$ Oscillator strength. ${ }^{c} \mathrm{H}$ stands for HOMO and L stands for LUMO. Only the main configurations are presented (CI coefficients $>0.1$ ). ${ }^{d}$ CI coefficients are in absolute values.

shift of fluorescence emission spectrum can be observed. Afterwards, coordination with metal ions will reduce electron donating ability of IIP, as well as the conjugation extent. The electron negativity of carbonyl returned with electron density of -0.14 e. Moreover, the energy gap between HOMO and LUMO is usually proportional to conjugation extent of molecule. ${ }^{40}$ The energy gap of $\mathrm{Cu}$-IIP-fluorescein is -0.1328 a.u.; after coordinating with copper ions, the energy gap are -0.1293 a.u. ( $\alpha$ electron) and -0.1142 a.u. ( $\beta$ electron), which are both declined. On the basis of these results, we conclude that the PET and ICT function together in sensing process of IIP-fluorescein nano probes.

The fluorescence quantum yields of probes were calculated as eqn (1). Fluorescein was chosen as standard substance with fluorescence quantum yield of $95 \%$ (in $0.1 \mathrm{M} \mathrm{NaOH}$ solution). The results for Cu-IIP-fluorescein, Zn-IIP-fluorescein, Cr-IIPfluorescein and NIP-fluorescein were $0.861,0.801,0.820$ and 0.745 , respectively. The slight decrease of quantum yields comparing with fluorescein is due to the absorbance of IIP or NIP cannot transform into fluorescence radiation. The quantum yields of M-IIP-fluorescein are overall higher than NIP-fluorescein. It reveals the imprinting effect of metal ions, which increase regularity and rigidity of the molecular chain. The results demonstrate probes still have extremely high fluorescence quantum yields and might be applicable in high sensitive detection of metal ions. The details of absorbance and fluorescent peak area are given in Table $\mathrm{S} 2$ in the ESI. $\dagger$

$$
Y \frac{A}{F}=Y_{\mathrm{S}} \frac{A_{\mathrm{S}}}{Y_{\mathrm{S}}}
$$

where $Y$ and $Y_{\mathrm{S}}$ are the fluorescence quantum yields of probe and fluorescein. $A$ and $A_{\mathrm{S}}$ are the absorbance of probe and fluorescein. $F$ and $F_{\mathrm{S}}$ are the fluorescent area of probe and fluorescein.

\subsection{Sensitivity and detection of three fluorescent probes for} $\mathrm{Cu}^{2+}, \mathrm{Zn}^{2+}$ and $\mathrm{Cr}^{3+}$

In our previous study, PEI presented strong coordination effect with $\mathrm{Cu}^{2+}, \mathrm{Zn}^{2+}, \mathrm{Cr}^{3+}$ et al. due to its specific polymer structure and affinity of amino group. ${ }^{\mathbf{4 1}}$ The computational values in Fig. S6† also confirmed this result. Sensing of metal ions depends on the interaction between IIP and metal ions as mentioned above. The imprinting effects by different metal ions will enhance the selectivity to certain template ion. To evaluate the selectivity of this protocol, difference of fluorescence spectra for the three probes with each metal ion was compared. As indicated in Fig. 3, each probe reveals specific recognition to the relevant template metal ion. In contrast to significant quenching as observed for template metal ion, negligible signal response is observed for the other metal ions. A slight interference between copper ion and zinc ion is due to their similar complex structure and ion radius.

To further check the selectivity and resistance to interference by other metal ions, competition experiments were conducted in the presence of 1 equiv. of each interfering ion, with the subsequent addition of 1 equiv. of template metal ion. As shown in Fig. 4, the emission profile of the $\mathrm{Cu}$-IIP-fluorescein $/ \mathrm{Cu}^{2+}$, $\mathrm{Zn}$-IIP-fluorescein $/ \mathrm{Zn}^{2+}$ and Cr-IIP-fluorescein/ $/ \mathrm{Cr}^{3+}$ complex 

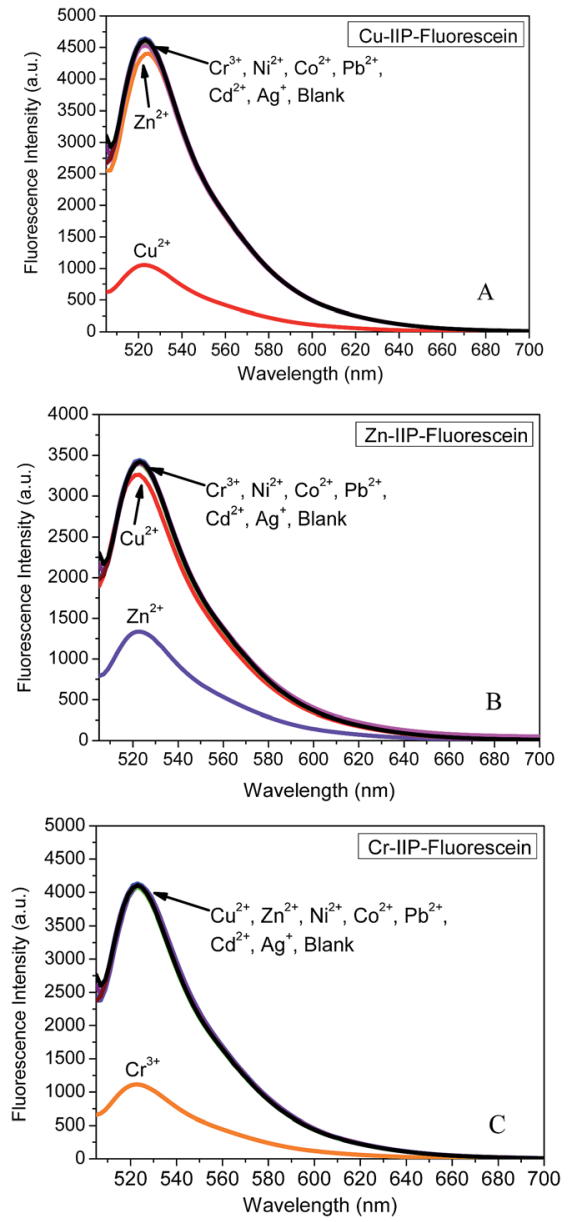

Fig. 3 Emission spectrum of IIP-fluorescein coordinating with each metal ion. (A) Cu-IIP-fluorescein, (B) Zn-IIP-fluorescein and (C) $\mathrm{Cr}$ IIP-fluorescein

are all unperturbed in the presence of these interfering ions, indicating the strongest affinity and selectivity for template metal ion. A reasonable explanation would be that the structure and dimension of imprinted sites in IIP only fit for template metal ion. The selectivities of three probes are independent and show specific affinity to each template metal ion. Thus the controllable selectivity of PEI fluorescent probe could be achieved, which promotes applications in recognition and extraction of certain target metal ions.

According to the above standard procedures and under the optimized assay conditions, different concentrations of $\mathrm{Cu}^{2+}$, $\mathrm{Zn}^{2+}$ or $\mathrm{Cr}^{3+}$ were added to the buffer and fluorescence emission intensity was measured to evaluate the sensitivity of the probes. As shown in Fig. 5, fluorescence emission intensity decreased gradually with the increase of template ion concentration. For further increasing of metal ions, the fluorescence quenching rates gradually stabilized and fluorescence intensity retained $21.5 \%, 27.0 \%$ and $24.9 \%$ for Cu-IIP-fluorescein, $\mathrm{Zn}$-IIP-fluorescein, Cr-IIP-fluorescein, respectively. The decrement fluorescence emission intensity was found to be linear with the concentration of $\mathrm{Cu}^{2+}, \mathrm{Zn}^{2+}$ or $\mathrm{Cr}^{3+}$ in the range of $6.65 \times 10^{-6}$ $1.17 \times 10^{-2} \mathrm{~g} \mathrm{~L}^{-1}, 9.17 \times 10^{-6}-8.03 \times 10^{-3} \mathrm{~g} \mathrm{~L}^{-1}$ and $8.39 \times$

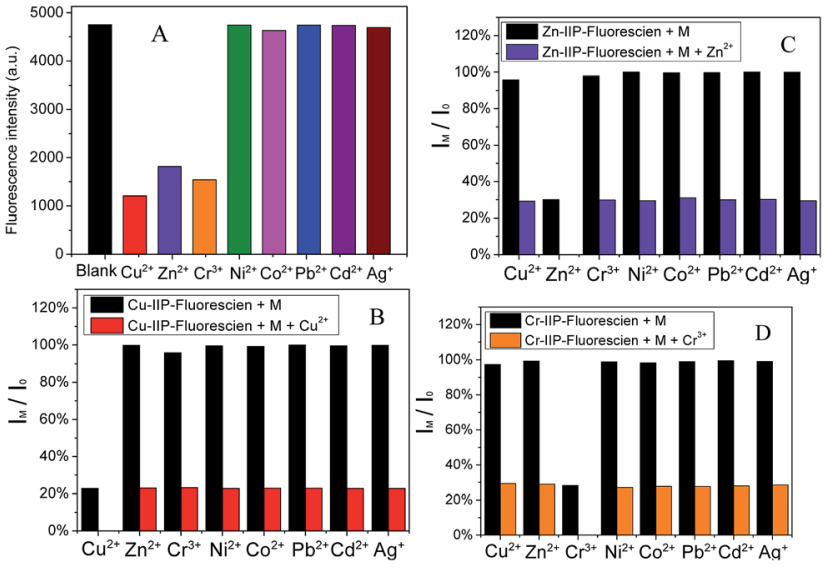

Fig. 4 (A) Fluorescence intensity histogram of NIP-fluorescein to each metal ion. ( $B, C$ and $D)$ Relative fluorescence intensity histogram of three nano-fluorescent probes to metal ions, respectively. Bars represent the final fluorescence intensity at $523 \mathrm{~nm}\left(I_{M}\right)$ over the original emission at $523 \mathrm{~nm}\left(I_{0}\right)$. Black bars represent the addition of $10 \mathrm{mg} \mathrm{L}^{-1}$ of metal ions to a $0.1 \mu \mathrm{M}$ solution of nano-fluorescent probe. Red, violet or orange bars represent the subsequent addition of $10 \mathrm{mg} \mathrm{L}^{-1}$ of $\mathrm{Cu}^{2+}, \mathrm{Zn}^{2+}$ or $\mathrm{Cr}^{3+}$ to the solution, respectively.

$10^{-6}-1.02 \times 10^{-2} \mathrm{~g} \mathrm{~L}^{-1}$. According to the standard deviation for the blank signal with 15 parallel measurements, limits of detection of $1.99 \times 10^{-6} \mathrm{~g} \mathrm{~L}^{-1}, 3.67 \times 10^{-6} \mathrm{~g} \mathrm{~L}^{-1}, 2.75 \times 10^{-6} \mathrm{~g}$
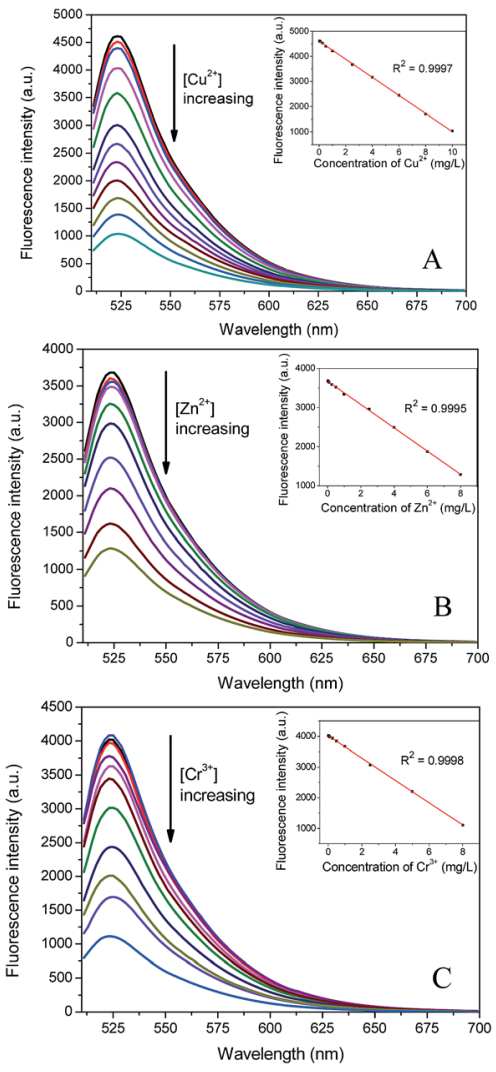

Fig. 5 Fluorescence spectra and titration curves of the nano-fluorescent probes to template metal ions. (A) Cu-IIP-fluorescein. (B) ZnIIP-fluorescein and (C) $\mathrm{Cr}$-IIP-fluorescein. 
$\mathrm{L}^{-1}$ for $\mathrm{Cu}^{2+}, \mathrm{Zn}^{2+}$ and $\mathrm{Cr}^{3+}$ were estimated by 3 times the standard deviation rule. The World Health Organization (WHO) regulate the maximum allowable levels of $\mathrm{Cu}^{2+}, \mathrm{Zn}^{2+}$ and $\mathrm{Cr}^{3+}$ in drinking water to be $1.0 \times 10^{-3} \mathrm{~g} \mathrm{~L}^{-1}, 1.0 \times 10^{-3} \mathrm{~g} \mathrm{~L}^{-1}$ and $5 \times$ $10^{-5} \mathrm{~g} \mathrm{~L}^{-1}$, respectively. The nano-fluorescent probes present the wide linearity range in detection. Moreover, the limit of detection is appropriate to monitor the metal ions changes in natural water or contaminated water.

\subsection{Application of fluorescent probes in environmental water sample}

To verify the practical application of the proposed method, several environmental water samples spiked with metal ions were measured using the proposed method and flame atomic absorption spectrometry (FAAS). The environmental water samples used in the study were tap water and river water. The river water samples were filtered by qualitative filter paper and then centrifuged for $20 \mathrm{~min}$ at $12000 \mathrm{rpm}$. The samples spiked with different concentrations of metal ions were detected according to the general procedure with three replicates. The results were summarized in Table 2 and showed good agreement with the values determined by FAAS. The mean deviation of fluorescent probes and FAAS comparing with truth value are $1.52 \%$ and $1.71 \%$, respectively. It is also notable that both methods prove precise selectivity for target ion in the metal mixture. The results reveal the present probes could be excellently applied in environmental detection.

As mentioned above, the probes reveal excellent selectivity and precision for template metal ions. Moreover, the probes also show potential in removing metal ions due to their large adsorption capacity and affinity. To evaluate adsorption and removal ability of the probes, river water samples spiked with mixed metal ions $\left(\mathrm{Cu}^{2+}, \mathrm{Zn}^{2+}, \mathrm{Cr}^{3+}, \mathrm{Ni}^{2+}, \mathrm{Co}^{2+}, \mathrm{Pb}^{2+}, \mathrm{Cd}^{2+}\right.$ and $\mathrm{Ag}^{+}$with each of $\left.1 \mathrm{mg} \mathrm{L}^{-1}\right)$ were measured as Fig. S7A. $\dagger$ The template metal ions $\left(\mathrm{Cu}^{2+}, \mathrm{Zn}^{2+}\right.$ or $\left.\mathrm{Cr}^{3+}\right)$ can be selectively removed by relevant probe. After one time of adsorption by each probe, the removal rate could reach $93.4 \%, 87.9 \%$ and $91.7 \%$. The adsorbed metal ion could be eluted with hydrochloric acid or ethylene diamine tetraacetic acid (EDTA), result in recover rate of $84.6 \%, 79.3 \%$ and $80.4 \%$ for $\mathrm{Cu}^{2+}, \mathrm{Zn}^{2+}$ and $\mathrm{Cr}^{3+}$. The removal rates of probes dependence with concentration of metal ions were also conducted as Fig. S7B. $\uparrow$ The concentration of metal ions is proposed to be less than $10 \mathrm{mg} \mathrm{L}^{-1}$ with effective removal by $0.1 \mu \mathrm{M}$ of probes.

The anti-interference ability of fluorescent probe is an essential target in application. Several common pollutants were added into river water (spiked with $\mathrm{Cu}^{2+}, \mathrm{Zn}^{2+}$ and $\mathrm{Cr}^{3+}$ each of $1 \mathrm{mg} \mathrm{L}^{-1}$ ) to simulate concrete situations of water pollution. The detection and removal rate of probes for template metal ions were measured as Table $\mathrm{S} 3 . \dagger$ The results indicate suspended solids $\left(\mathrm{KAl}\left(\mathrm{SO}_{4}\right)_{2} \cdot 12 \mathrm{H}_{2} \mathrm{O}\right)$ and colouring agent $\left(\mathrm{KMnO}_{4}\right)$ will cause severe disturbance for determination. The reasonable explanation would be that light scattering or absorption by the pollutants will influence the intensity of fluorescence emission. The other common organic and inorganic pollutants will cause neglectable influence to determination. However, the removal rates for metal ions remain very high in the presence of those common pollutants, indicating the excellent performance on heavy metal ion treatment in environment water.

\subsection{Reversibility and reusability of fluorescent probes}

The probe has a very low fluorescence emission after being used to monitor metal ions. However, when it is treated with high concentrations of hydrochloric acid or EDTA and repeatedly rinsed with DI-water, the metal ions are eluted and its fluorescence intensity could be recovered. After five times of reuse and regeneration, the average intensity of three probes still remain as high as $87.3 \%$ of initial intensity. The fluorescence intensity and adsorption capacity of reused probes by different eluent were measured as shown in Fig. S8. $\dagger$ The decrease of fluorescence intensity and adsorption capacity with eluting by EDTA is due to residual metal ion and run off of the probes. However, a larger decrease of fluorescence intensity and increase of adsorption capacity are observed with eluting by hydrochloric acid. It can be explained that the amido bond between fluorescein and IIP is partly broken by hydrochloric acid, which

Table 2 Determination of $\mathrm{Cu}^{2+}, \mathrm{Zn}^{2+}$ and $\mathrm{Cr}^{3+}$ in environment water samples using the fluorescent probes and FAAS

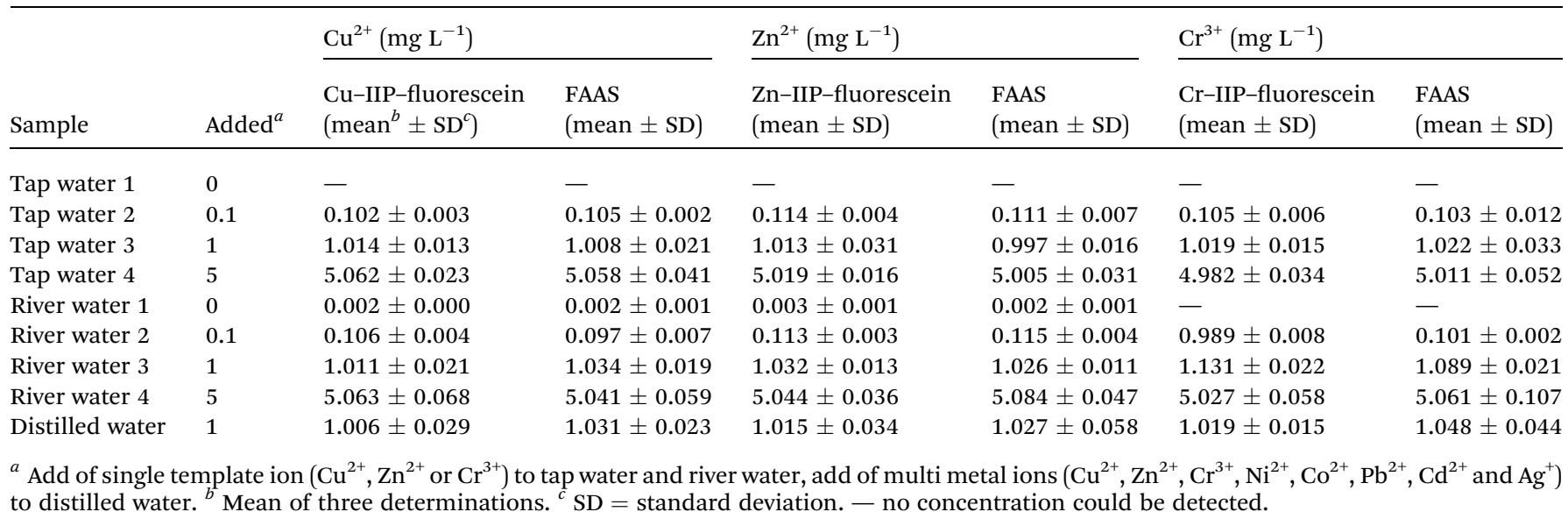


causes run off of fluorescein moieties. So it is essential to avoid application of probes under strong acidic condition.

\section{Conclusions}

In summary, three prototypes based on polyethyleneimine of highly sensitive and selective nano-fluorescent probes for $\mathrm{Cu}^{2+}$, $\mathrm{Zn}^{2+}$ and $\mathrm{Cr}^{3+}$ were successfully obtained. The selectivity differentiation of polyethyleneimine could be achieved through imprinting process with several kinds of metal ions. Each probe reveals specific selectivity and recognition to the relevant template metal ion over seven other common metal ions. The metal ion imprinted fluorescent probes exhibit a large linear detection range, as well as the strong adsorption capacity for $\mathrm{Cu}^{2+}, \mathrm{Zn}^{2+}$ and $\mathrm{Cr}^{3+}$. The measurement accuracy of three probes was also confirmed by comparison with AAS. The probes have been tested to detect and remove $\mathrm{Cu}^{2+}, \mathrm{Zn}^{2+}$ and $\mathrm{Cr}^{3+}$ in polluted tap water and river water, demonstrating their potential application as practical environmental probes. The sensing mechanism for metal ions was investigated with Frontier molecular orbital and electron density distribution by Gaussian 09. It was found that the PET and ICT function together in sensing process of IIP-fluorescein nano probes process. Furthermore, the affinity between polyethyleneimine and different metal ions was verified by simulation and experiment, revealing potential direction of selectivity differentiation of PEI. The results might propose a method for developing and studying other fluorescent probes with similar systems (containing amino groups, mercapto groups or cyano groups).

\section{Acknowledgements}

This work was financially supported by the National Natural Science Foundation of China (21447008), Science and Technology Program of Shenzhen and Guangdong (CXZZ20140902141250786, 2014A010105004).

\section{References}

1 G. Zhao, J. Li, X. Ren, C. Chen and X. Wang, Environ. Sci. Technol., 2011, 45, 10454-10462.

2 L. J. Xu and J. L. Wang, Environ. Sci. Technol., 2012, 46, 10145-10153.

3 V. P. Utgikar, N. Chaudhary, A. Koeniger, H. H. Tabak, J. R. Haines and R. Govind, Water Res., 2004, 38, 3651-3658.

4 H. Hasegawa, I. M. Rahman, M. Nakano, Z. A. Begum, Y. Egawa, T. Maki, Y. Furusho and S. Mizutani, Water Res., 2011, 45, 4844-4854.

5 K. Ha Na, G. Zhiqian, Z. Weihong, Y. Juyoung and T. He, Chem. Soc. Rev., 2011, 40, 79-93.

6 L. Xiaohua, G. Xinghui, S. Wen and M. Huimin, Chem. Rev., 2014, 114, 590-659.

7 W. Yong-Xiang, Z. Xiao-Bing, L. Jun-Bin, Z. Cui-Cui, L. Hao, M. Guo-Jiang, Z. Li-Yi, T. Weihong and Y. Ru-Qin, Anal. Chem., 2014, 86, 10389-10396.
8 P. J. Jiang, L. Z. Chen, J. Lin, Q. Liu, J. Ding, X. Gao and Z. J. Guo, Chem. Commun., 2002, 1424-1425, DOI: 10.1039/ b202976f.

9 Y. Xie, Y. Ding, X. Li, C. Wang, J. P. Hill, K. Ariga, W. Zhang and W. Zhu, Chem. Commun., 2012, 48, 11513-11515.

10 H. S. Jung, P. S. Kwon, J. W. Lee, J. I. Kim, C. S. Hong, J. W. Kim, S. Yan, J. Y. Lee, J. H. Lee, T. Joo and J. S. Kim, J. Am. Chem. Soc., 2009, 131, 2008-2012.

11 J. Chan, S. C. Dodani and C. J. Chang, Nat. Chem., 2012, 4, 973-984.

12 T. Baumgart, G. Hunt, E. R. Farkas, W. W. Webb and G. W. Feigenson, Biochim. Biophys. Acta, Biomembr., 2007, 1768, 2182-2194.

13 X. Michalet, F. F. Pinaud, L. A. Bentolila, J. M. Tsay, S. Doose, J. J. Li, G. Sundaresan, A. M. Wu, S. S. Gambhir and S. Weiss, Science, 2005, 307, 538-544.

14 I. L. Medintz, H. T. Uyeda, E. R. Goldman and H. Mattoussi, Nat. Mater., 2005, 4, 435-446.

15 B. N. G. Giepmans, S. R. Adams, M. H. Ellisman and R. Y. Tsien, Science, 2006, 312, 217-224.

16 W. Wei, X. Liu, P. Sun, X. Wang, H. Zhu, M. Hong, Z.-W. Mao and J. Zhao, Environ. Sci. Technol., 2014, 48, 3363-3371.

17 W. Wei, T. Zhu, Y. Wang, H. Yang, Z. Hao, P. R. Chen and J. Zhao, Chem. Sci., 2012, 3, 1780-1784.

18 X. Cui, L. Zhu, J. Wu, Y. Hou, P. Wang, Z. Wang and M. Yang, Biosens. Bioelectron., 2015, 63, 506-512.

19 X. Chen, Y. Zhou, X. J. Peng and J. Yoon, Chem. Soc. Rev., 2010, 39, 2120-2135.

20 M. Bruchez, M. Moronne, P. Gin, S. Weiss and A. P. Alivisatos, Science, 1998, 281, 2013-2016.

21 C. Wang, H. Tao, L. Cheng and Z. Liu, Biomaterials, 2011, 32, 6145-6154.

22 J. S. Yang and T. M. Swager, J. Am. Chem. Soc., 1998, 120, 11864-11873.

23 L. Mu, W. Shi, J. C. Chang and S.-T. Lee, Nano Lett., 2008, 8, 104-109.

24 T. P. Rao, R. Kala and S. Daniel, Anal. Chim. Acta, 2006, 578, 105-116.

25 M. Behbahani, A. Bagheri, M. Taghizadeh, M. Salarian, O. Sadeghi, L. Adlnasab and K. Jalali, Food Chem., 2013, 138, 2050-2056.

26 S. Dai, M. C. Burleigh, Y. H. Ju, H. J. Gao, J. S. Lin, S. J. Pennycook, C. E. Barnes and Z. L. Xue, J. Am. Chem. Soc., 2000, 122, 992-993.

27 O. Guney and F. C. Cebeci, J. Appl. Polym. Sci., 2010, 117, 2373-2379.

28 L. Uzun, R. Uzek, S. Senel, R. Say and A. Denizli, Mater. Sci. Eng., C, 2013, 33, 3432-3439.

29 M.-R. Chao, C.-W. Hu and J.-L. Chen, Microchim. Acta, 2014, 181, 1085-1091.

30 O. V. Chumakova, A. V. Liopo and V. G. Andreev, Cancer Lett., 2008, 261, 215-225.

31 S. Kagaya, H. Miyazaki, Y. Inoue, T. Kato, H. Yanai, W. Kamichatani, T. Kajiwara, M. Saito and K. Tohda, J. Hazard. Mater., 2012, 203-204, 370-373.

32 B. Gao, F. An and Y. Zhu, Polymer, 2007, 48, 2288-2297. 
33 J. Jia, A. Wu and S. Luan, Phys. Chem. Chem. Phys., 2014, 16, 16158-16165.

34 M. J. Frisch, G. W. Trucks and H. B. Schlegel, Gaussian 09 revision C.01, Gaussian Inc, Wallingford, CT, 2010.

35 B. Baleur, Principles of Fluorescence Spectroscopy, Wiley-VCH Verlag GmbH, New York, 2001.

36 J. Shaomin, Y. Jun, Y. Qing, L. Shasha, C. Maodu and Z. Jianzhang, J. Org. Chem., 2009, 74, 4855-4865.

37 H. Feng, C. Lina, L. Xiaofen, J. Shaomin, L. Shasha, Z. Fuke, W. Yubo, H. Keli, Z. Jianzhang and T. D. James, J. Org. Chem., 2009, 74, 1333-1336.
38 G. J. Zhao, J. Y. Liu, L. C. Zhou and K. L. Han, J. Phys. Chem. $B, 2007,111,8940-8945$.

39 S. Kenichiro, N. Manabu, Z. Kiyoshi, N. Shinkoh and S. Hiroshi, J. Phys. Chem. A, 2009, 113, 8213-8220.

40 Y. M. Jiang, A Relationship Between Electrical Conductivity and the Hbmo-Labmo Energy Gap in Doped Poly, University of Southern Mississippi, 1993.

41 J. Jia, A. Wu and S. Luan, Colloids Surf., A, 2014, 449, 1-7. 